\section{BILIRUBIN IN THE BRAIN: NEUROTOXIC EFFECTS, THERAPEUTIC PROMISES AND REGIONAL VULNERABILITY}

A.R. Vaz, S.L. Silva, A.S. Falcão, A. Barateiro, A. Fernandes, M.A. Brito, R. Silva, D. Brites

Research Institute for Medicines and Pharmaceutical Sciences (iMed.UL/CPM), Faculdade de Farmácia, University of Lisbon, Lisbon, Portugal

Elevated levels of free bilirubin in the brain triggered by increased concentrations of unconjugated bilirubin (UCB) may cause adverse neurological outcomes. We aimed to evaluate: (i)the effects of UCB in neuronal apoptosis, oxidative stress and P-JNK1/2 expression; (ii) if UCB damages the neuritic tree and whether glycoursodeoxycholic acid (GUDCA), IL-10 or L-NAME are protective; (iii)the brain regional susceptibility to UCB-induced dendritic disruption.

Rat cortical neurons with 3 days in vitro (DIV) were incubated with 50 microM UCB plus 100 microM HSA. After 4h, we evaluated apoptosis (caspase-3 activity), P-JNK1/2 and nNOS expression (Western blot) and nitrites (Griess reagent). Following 24h, neurite arborization (MAP-2 labeling) was evaluated. Cells were also incubated with 50 microM GUDCA, $10 \mathrm{ng} / \mathrm{mL}$ IL-10 or 100 microM L-NAME prior to UCB exposure. Neurons from hippocampus and cerebellum at 3 DIV were also exposed to UCB (24h) to assess neuritic arborization.

UCB increased caspase- 3 activity (1.5-fold, $p<$ $0.01), P-J N K 1 / 2$ (1.3-fold, $p<0.01)$ and nNOS (1.2fold, $p<0.01$ ), as well as NO production (1.7-fold, $p<$ $0.01)$. Moreover, UCB decreased neurite extension (0.8-fold, $p<0.05)$ and ramification $(0.5$-fold, $p<$ 0.05 ), effects that were prevented by GUDCA ( $p<$ 0.05 vs UCB), IL-10 and L-NAME (extension, $p<$ 0.05 ; ramification, $p<0.01$ vs UCB). Hippocampus revealed the utmost reduction in neurite ramification $(46 \%, p<0.01)$ and extension (28\%, p< 0.05).

Neuronal dysfunction by UCB comprises several intracellular pathways involving oxidative injury and disruption of neurite arborization. Anti-oxidant and/or anti-inflammatory compounds prevent UCBdamage to neuronal network. Hippocampus reveals the higher neurotoxicty by UCB. Funding:FCTPTDC/SAU-NEU/64385/2006.

\section{NEUROPROTECTION WITH RHEPO IN CYANTOIC NEONATAL MICE}

\author{
H.H. Odland ${ }^{1}$, A.L. Aasland ${ }^{1}$, H. Carlsen², \\ R. Blomhoff ${ }^{2}$, O.D. Saugstad ${ }^{1}$
}

${ }^{1}$ Department of Pediatric Research, ${ }^{2}$ Department of Nutrition, Oslo University Hospital, Oslo, Norway

Background and aims: Erythropoietin is a known neuroprotective agent in the presence of EpoR and CD131. Hypoxia may induce EpoR and increase rhEPO binding in peripheral tissue. We wanted to study whether rhEPO is protective in mice preconditioned with hypoxemia in a cyanosis model.

Methods: 50 neonatal transgenic cerebral luciferase-NFkB reporter mice from 7 litters were used. At the day of birth 3 litters and mothers were kept in a hypoxic environment $\left(\mathrm{FiO}_{2}=0.12\right)$, while 4 litters were kept under normoxic conditions. At day seven, five hypoxemic mice and 14 normoxemic mice pre-treated with rhEPO, underwent selective occlusion of the left carotic artery and hypoxia (4\% oxygen) together with 15 hypoxemic and 15 normoxemic mice. Signals were measured from the ex vivo left hemisphere as luminescence (logtransformed), and compared to normoxemia + rhEPO mice that were expected to have the least neuronal damage. Weight was used as a covariate. Values are estimated means \pm SEM based on weight $3.77 \mathrm{~g}$.

Results: Normoxemia + rhEPO and hypoxemia was not different $(7.61 \pm 0.04$ vs. $7.67 \pm 0.04)$, while normoxia alone, and hypoxemia + rhEPO had significantly higher expression of NFKB than normoxemia + rhEPO $(7.91 \pm 0.04 ; 7.88 \pm 0.06$ vs. $7.61 \pm 0.04, p<0.05)$.

Conclusion: Erythropoietin increases NFkB expression in hypoxemic preconditioned mice comparable to the increase of NFKB in not treated normoxemic mice after cerebral insult. This indicates that rhEPO may be detrimental in hypoxemic mice and warrants further studies before using rhEPO as a presumptive neuroprotective agent in cyanotic individuals. 